\title{
Research on Quick Visualization Analysis Model of Power Big Data Based on 3D Scene
}

\author{
MA JianWei ${ }^{1, a}$, WAN HuiJiang ${ }^{2, b}$, LIANG Ling ${ }^{3, c}$, JIANG ZaiNeng ${ }^{4, d}$ \\ ${ }^{1,2,3,4}$ Guizhou Power Grid Co., Ltd., power dispatching control center, Guiyang, Guizhou 550002 \\ China \\ at425054771@qq.com; b408438504@qq.com; c361550717@qq.com ; d991980142@qq.com
}

Keywords: visualization; virtual reality; big data; supercomputing

\begin{abstract}
The ideas based on integrating power big data and visualization, this paper firstly puts forward quick visualization analysis model of power big data based on 3D scene, it designs several levels to realize abstraction in business, engine, computation, control and storage, it integrates the separated power data storage, data mining and analysis, data visualization in the past into the same platform. Secondly, as for real -time rendering problem of large-scale 3D scene of model, this paper respectively visibility exclusion method with high efficiency and quick rendering method with multi-resolution ratio to realize quick construction and rendering in $3 \mathrm{D}$ virtual reality scene of power system under big data environment.
\end{abstract}

\section{Introduction}

Visualization method is the important measure always used to represent and analyze data. Research indicates there is above obtained $80 \%$ information from outside comes from vision system. When data is represented by intuitive graphical form, users can instantly know the hidden information of data expressed by graph and quickly convert it into knowledge. In big data times, because data and data dimension is drastically increasing, the traditional vision methods can no longer intuitive. In view of traditional visualization methods are in fact $2 \mathrm{D}$ projection of $\mathrm{N}$-dimensional data, as for big data, traditional dimension flattening visualization method makes excessive dimensions compressed to the plain, it lost space information of dimension itself and can not effectively represent data characteristics. The difference of power big data from other kind big data lies in that it has typical space topology structure, dispersed data is generally corresponds to power equipment of 3D space. This paper uses 3D scene as base to implement data analysis and visualization work.

\section{Quick visualization analysis model of power big data based on 3D scene}

\section{A. GPU architecture and graph rendering pipe line}

Vertex transformation: vertex multiplies local transformational matrix, which makes model rotate or stretch, and then it multiplies transformational matrix, which moves model from local coordinate system to world coordinate system. Afterwards, it multiplies projection transformational matrix to rotate and move orthotropic world coordinate system, which is used to simulate viewpoint and perspective effect, in the end, it multiplies viewpoint transformational matrix, and makes coordinate normalization, which is transformed to the uniform coordinate system of designated window. Figure 1 indicates the process of world coordinate system multiplies projection matrix transformed into visual space. 


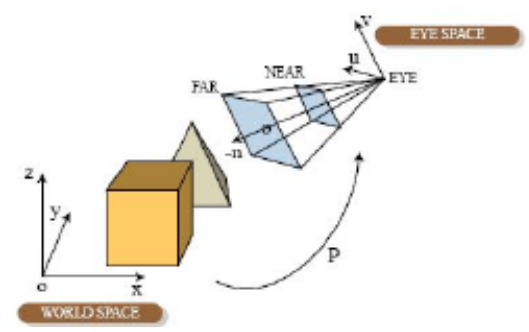

Figure 1 Vertex transformation of 3D graph rendering pipe line

From graph 2 it can be seen that control circuit area of CPU is larger than GPU, but its computational element is smaller than GPU and it has no detailed hierarchical division. In addition, GPU pertinently designs expert optimization on vector, matrix and floating-point calculation, and it has high concurrency characteristic. So the compete separation times of CPU and GPU is ended, it appears concept of using GPU to implement universal calculation, which makes CPU and GPU together process calculation tasks, which is GPGPU. Expert GPGPU programming language appears which are CUDAC and OpenCL.

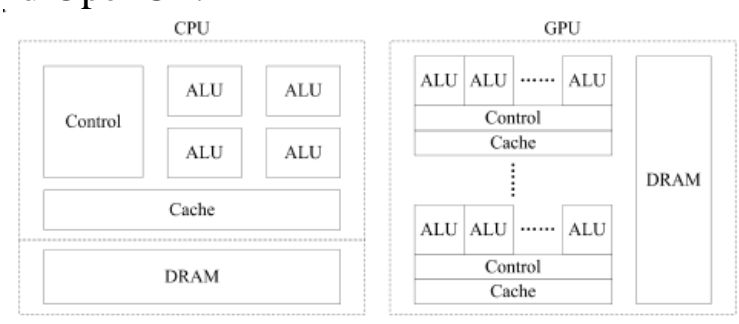

Figure 2 CPU architecture and GPU architecture

B. Data structure of scene graph

BSP tree. BSP tree is one kind of structure with stronger specificity, it can be only used in visibility search of indoors scene on roaming (it is limited in certain dimension codomain). BSP tree makes 2-value division on space; it usually adopts any one piece of axis of bounding box as division plain. As for background of given object at present, its axis plain divides space into 2 parts, which defines one part is in the back of this object and the other part is in front of this object. As for divided subspace, it follows this definition and makes further division, then all the subspace and all objects have relative position gate. (2) Quaternary tree. It is one kind of structure with stronger specificity; it is only used in altitude data such as terrain. Quaternary tree is the expansion of binary tree in space, because it is usually used in terrain, so it has better node balance, search cost is relatively even, it is unnecessary to generate hierarchical model with multi-resolution ratio on further optimization. (3) Octree, it is one kind of common structure, it is only used in large-scale scene. Octree is the expansion of binary tree in space, its root node represents the size of the whole bounding box, and it evenly divides it into 8 subspaces.
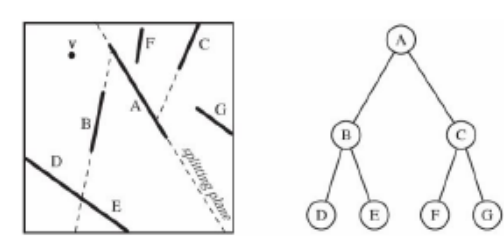

Figure 3 BSP tree
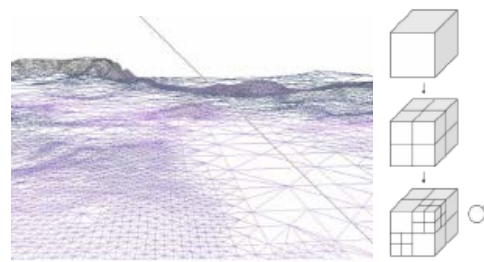

Figure 4 Quaternary tree Figure 5 Octree

Table 1 Applicability, advantage and disadvantage of usual tree scene graph

\begin{tabular}{|c|c|c|c|}
\hline structure & applicable scene & advantages & disadvatages \\
\hline BSPtree & indoors scene & $\begin{array}{c}\text { high efficiency on } \\
\text { operation }\end{array}$ & $\begin{array}{c}\text { complicate architecure } \\
\text { algorithm }\end{array}$ \\
\hline quaternary tree & $\begin{array}{c}\text { altitude scene such as } \\
\text { terrain }\end{array}$ & $\begin{array}{c}\text { it is favorable for LOD } \\
\text { optimization }\end{array}$ & bad univeral property \\
\hline octree & $\begin{array}{c}\text { outdoors large-scale } \\
\text { scene }\end{array}$ & good univeral property & bad node balance \\
\hline
\end{tabular}


Halton sequence is the even random method of value method (typical Monte Carlo method), it is used to generate even and random point. The basic principles of Halton sequence is as follows: (1) choose one prime number as cardinal number, (2) as for the given ordinal N0, convert N0 into hexadecimal number N1; (3) move forward of radix point of $\mathrm{N} 1$ and it is transformed into decimal number smaller than 1; (4) convert N2 into decimal number N3. It applies $[0,1]$ range of N3 to implement interpolation, which indicates even randomness.

Figure 6 Distribution of pseudo-random number and sequence random number in 2D plain

C. Model simplification algorithm based on LOD weight function

On simplifying model, it firstly simplifies unimportant $\mathrm{E}$ and gradually transits to important $\mathrm{E}$. Obviously, how to determine the importance degree of $E$, which means how to quantify the importance of $E$ is the primary problem, H.Hoppe puts forward energy function idea, formula (1) defines approximation degree of simplification model and original model, weight change according to vertex quantity change and one corrected value. Surface reduction effect of energy function method is very ideal, it is excellent in keeping realness, meanwhile, the corresponding algorithm is relatively complicated and implementation efficiency is very low, so it can not be applied in quick real-time optimization in large-scale scene.

$$
E(K, V)=E_{\text {dist }}(K, V)+E_{\text {rep }}(K, V)+E_{\text {spring }}(K, V)
$$

The reasons why energy function formula and energy function algorithm of Hoppe is so complicated lie in that it firstly considers universal property, it takes effort to design universal surface reduction function for all the models, so it has good calculation effect and large calculation quantity, it can not achieve requirement of real-time visualization analysis system. In the actual 3D virtual reality scene application, in fact, model pattern in designated scene will not have big difference, for example, building scene is almost regular square model; power equipment scene is almost basic building model and equipment with ring structure. Considering from this point, we can design expert quantized function, so it can reduce complication caused by universal property and accelerate 3D scene rendering.

\section{Application of power big data quick visualization analysis based on $3 \mathrm{D}$ scene in power transmission industry}

Start from perspective of information technology, it represents full-scene of 3D, combines with virtual reality and information integration technology to construct visualization platform of grid 3D space, it also combines with key technologies such as modeling, scene simulation, information integration, visualization intersection and polymorphic calculation etc to implement visualization representation, innovatively puts forward engineering construction idea of 3D visualization information platform in city grid space, so it further enhances safe operation level of grid, increases controllable , capable of controlling and controlling capacity of power transmission state, really realizes $3 \mathrm{D}$ visualization information platform construction in city grid space.

A. Auxiliary design and design application of power transmission line planning

It uses vast DEM data with high precision, image data with high resolution ratio and 3D power equipment model to make full-scene simulation on the whole transmission line in computer, so that it realizes the actual realization on surrounding environment on this transmission line. This design department can realize transmission line planning and space analysis in the virtual 3D visualization full scene, makes transmission line more reasonable and so it achieves aim of optimizing line and reducing cost. $3 \mathrm{D}$ visualization technology can meanwhile greatly reduce investigation tasks in the field, reduce positive effect, strengthen environmental-protection awareness and intuitively restores 3D terrain.

B. Visualization application of line and pole

There are plenty of circuits and poles in transmission line, and architecture model accounts for most part, line direction and pole distribution have close relations with terrain. As for this point, 3D visualization technology applies elevation data, image data and vector data to make 3D map, on this basis of restoring real site scene, it provides plenty of induction poles and ranking methods, after 
pole induction, it can automatically adjust pole height and form right according to terrain elevation data, and it can automatically calculate pole direction and sag.

C.Management and application of transmission line equipment

3D full-scene visualization technology makes high-precision modeling and simulation on grid facility and equipment involved by right, and it can realize quick view on 3D data. Meanwhile, it can integrate nature information of rich power equipment, including basic geography information, equipment information, operation state information, natural environment information etc as well as multi-media information such as video and photos etc to reduce tasks in the field, so that it can increase management efficiency and realize intelligent management of transmission line engineering.

D. Management and application of transmission line safety production

It uses 3D full-scene visualization technology to quickly and intuitively learn trend of transmission line. Line channel of transmission line has long distance, channel has complicated geography environment, it can break through vision limitation of patrol personnel of line through platform represented by full-scene visualization technology, it can complete maintenance tasks such as real-time monitoring as well as trouble check and grid monitoring etc on many transmission lines. Therefore, 3D visualization technology can clearly reflect 3D real conditions in the outside, so it further realizes 3D visualization management on transmission line.

E. Safe operation and maintenance application of transmission line

In the whole right, due to effect of external factors such as temperature, humidity etc, of course we can not eliminate problems such as some external forces destroy pole and cause position deviation etc. Obviously, the above factors have great safety hidden danger on transmission line. Therefore, in the operation and maintenance process on the whole transmission line, it needs to use 3D full-scene technology to make analysis on these factors. Firstly, it calculates effect of the whole transmission line in its terrain on the whole line, so that personnel in line implementation find out these hidden safety problems. Therefore, adopting line terrain change detection of 3D visualization technology has reality significance on safe operation of transmission line. For example, orthographic image transformation and perspective projection transformation respectively corresponds to different projection matrix, switching projection matrix just needs to dispatch corresponding OpenGLAPI. The following is the 3D parallel coordinate diagram drawn by orthographic image transformation from different perspectives, of which the abnormal data uses red to plot point easy for identification. When it implements orthographic projection transformation along $-\mathrm{z}$ axis direction, the diagram degenerates into 2D parallel coordinate diagram.

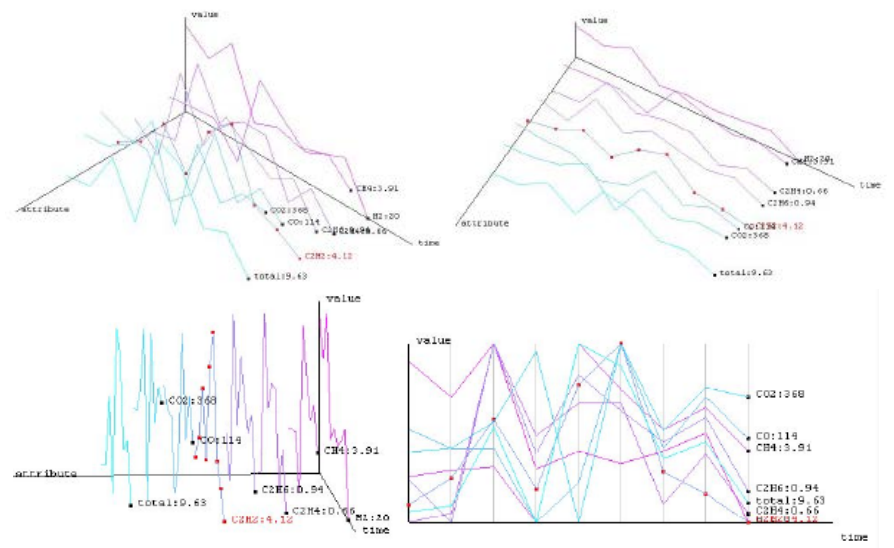

Figure 7 3D parallel coordinate diagram

F. High integration between space information and business data

The business data in the past is mainly indicated in table or words description, it is relatively insufficient in data extensity and objectivity indication. While 3D full-scene visualization technology can realize high integration and good effect by establishing correlation space information and business data. Under the macro watching background, we can check space position of grid equipment and its corresponding business data. Under the micro watching background, we can check its corresponding business data information by clicking high-precision model of 
corresponding equipment, so that it can really realize coordination work of visualization and visual management.

\section{Conclusions}

This paper designs visualization analysis model of power big data and integrates the above-mentioned scene with data, expecting to realize visualization analysis platform of systematic power big data. The model designed by this topic can make visualization and data analysis work integrates in the big data architecture without implementing it separately, which solves difficulty in mutual separation in data and resources, integration. As for the most important subsystem-visualization engine, it puts forward 2 kinds of quick rendering methods. As for large-scale scene real-time rendering problem, this paper designs graph data reduction method of 2 levels. Firstly is shelter and elimination of object level, it is realized based on octree and Halton random sequence, test result indicates its accuracy and effectiveness is very high, secondly is multi-resolution ratio drawing method within object element level. Integration between data visualization analysis and 3D scene is one development direction for power big data visualization technology. The full-scene state and nature of power big data determines visualization way based on 3D scene is helpful to enhance data comprehension and analysis processing result, and it has important significance for data visualization research.

\section{References}

[1]Cao Junwei, Yuan Zhongda, Ming Yangyang, Zhang Huaying. Overview to Energy Internet and Big Data Analysis Technology [J]. Technology of Southern Power Grid, 2015, 11:1-12.

[2]Wang Quanqiang, Liu Min, Cheng Li. Research on Power Big Data of Intelligent Grid [J]. Electronic Technology and Software Engineering, 2016, 01:181-182.

[3] Zhang Jianye, Liu Kui, Zhang Jianguang, Liu Yongting, Zhou Yuelin. Research and Realization on Visualization Technology of Grid Big Data [J]. Science Technology Innovation, 2017, 29:5-6.

[4]Qu Chaoyang, Xiong Zeyu, Yan Jia,Xin Peng,Qu Nan. 3D Full-scene Management Method of Power Big Data Based on Space Division [J]. Journal of North China Electric Power University (Natural Science Edition), 2016, 02:23-29.

[5]Zhang Yue, Liu Daowei, Chen Shuyong, Ma Shiying, Deng Chunyu, Shi Mengjie, Wu Xi. Visualization Design on Full-scene Safety Defense System of Big Grid Driven by Information [J]. Power Information and Communication Technology, 2016, 12:46-51. 